\title{
Quantidade de Participantes em Bate-papo Educacio- nal: um Modelo Baseado em Teoria de Filas
}

\author{
Number of Participants in Educational Chat: a Model Based on Queuing Theory \\ Edmilson Barcelos Rocha \\ Universidade Federal do Estado do \\ Rio de Janeiro - UNIRIO \\ Departamento de Informática Aplica- \\ da \\ Avenida Pasteur, 458 - Urca - Rio de \\ Janeiro \\ CEP: 22290-240 \\ edmilson.rocha@uniriotec.br \\ Mariano Pimentel \\ Universidade Federal do Estado do \\ Rio de Janeiro - UNIRIO \\ Departamento de Informática Aplica- \\ da \\ Avenida Pasteur, 458 - Urca - Rio de \\ Janeiro \\ CEP: 22290-240 \\ pimentel@uniriotec.br \\ Morganna Carmem Diniz \\ Universidade Federal do Estado do \\ Rio de Janeiro - UNIRIO \\ Departamento de Informática Aplica- \\ da \\ Avenida Pasteur, 458 - Urca - Rio de \\ Janeiro \\ CEP: 22290-240 \\ morganna@uniriotec.br
}

\begin{abstract}
Resumo Na educação dos dias atuais, a modalidade a distância é responsável por mais de 15\% das matrículas de graduação, sendo o fórum e o bate-papo os meios de conversação online mais utilizados. Essas informações motivaram a realização da pesquisa apresentada neste artigo cuja questão é: Quantos alunos devem participar de uma sessão bate-papo educacional de forma a obter um nivel satisfatório de participação dos alunos? O objetivo da presente pesquisa é estimar esse quantitativo de alunos por meio da elaboração de um modelo matemático que equacione o número de mensagens produzidas por aluno com a quantidade de alunos em uma sessão de bate-papo. Na elaboração do modelo, foi usado o método formal de modelagem com a aplicação da Teoria de Filas. Para avaliação do modelo, foram usados logs reais de bate-papos educacionais em que foram comparados os dados obtidos desses logs com as estimativas produzidas pelo modelo. As estimativas do modelo apresentaram-se dentro dos limites préestabelecidos para 95\% dos casos. Com isso, o modelo elaborado mostrou-se válido, o que possibilitou responder à questão da pesquisa.
\end{abstract}

Palavras-Chave: Bate-papo, Tamanho do Grupo, Participação Online, Modelagem Matemática, Sistemas de Fila

\begin{abstract}
In today's education, the distance mode represents more than 15\% of enrollment in graduate, forum and chat are the most used online conversations tools. It motived the research presented in this paper where the issue is: How many students will participate in an educational chat to ensure a satisfactory level of participation? The aim of this research is to estimate this number of students through the development of a mathematical model able to equating the number of messages produced by student with the number of students in a chat session. We used de formal modeling method based on Queuing Theory to build the model. Were used real logs of educational chats to evaluate the model comparing data obtained from these logs with the estimates produced by the model. The estimates produced by the model showed up within the preestabilished limits $95 \%$ of cases. Thus, the developed model proved to be valid, this allows to answer the research question.
\end{abstract}

Keywords: Chat, Group Size, Online Participation, Mathematical Modeling, Queuing Systems 



\section{Introdução: bate-papo na EAD}

A partir do ano 2000 ocorreu um grande crescimento da EAD no Brasil: de praticamente zero cursos a distância, em uma década, esta modalidade passou a ser responsável por quase $15 \%$ das matrículas na graduação, totalizando cerca de um milhão de estudantes [1]. A mídia mais utilizada em cursos a distância ainda é o material impresso: $87,3 \%$ das instituições utilizam material impresso, seguido pelo e-learning $(71,5 \%)$ e pelo vídeo $(51,7 \%)$ [2]. Mesmo com o material impresso ainda sendo a mídia mais utilizada, os recursos online têm ganhado cada vez mais espaço por favorecerem um novo paradigma de educação voltada para a interação. No Brasil, o bate-papo e o fórum são os meios de interação online mais utilizados na EAD, adotados por mais de $70 \%$ das instituições [2]. O grande avanço da EAD em nosso país e o fato do bate-papo ser um dos sistemas mais utilizados nesta modalidade motivaram a realização da pesquisa aqui apresentada.

Em estudos anteriores já foi constatado que o tamanho do grupo influencia a participação online $[3,4,5,6]$. A quantidade de usuários que devem participar de um bate-papo é encontrado na literatura de forma imprecisa. A imprecisão de quantos devem participar em um batepapo educacional se apresenta como o problema da pesquisa.

O objetivo desta pesquisa é estimar quantos alunos devem participar de uma sessão de bate-papo educacional de forma a garantir um nível de participação satisfatório. Para obter essa estimativa foi elaborado um modelo matemático que equaciona a quantidade de mensagens produzidas em função da quantidade de participantes. Até agora não foi encontrado na literatura um modelo que equacione a quantidade de mensagens com a quantidade de participantes, sendo este modelo o artefato objetivado como produto desta pesquisa.

Para desenvolver o modelo de participação em batepapo educacional, foi usado o método formal de modelagem de sistemas com aplicação da Teoria de Filas [7]. A avaliação do modelo proposto foi feita por meio de comparação das estimativas produzidas pelo modelo com dados obtidos de logs reais de bate-papo.

Esse artigo encontra-se estruturado nas seguintes seções. A questão de pesquisa - quantos alunos devem participar de um bate-papo educacional? - é discutida e formalizada na seção 2 . Na seção 3 são descritas as atividades que caracterizam a participação em sessões de bate-papo. A metodologia aplicada para o desenvolvimento do modelo da participação em bate-papo educacional é discutida na seção 4. Em seguida, na seção 5 são discutidos os resultados da avaliação do modelo elabora- do. Com o modelo validado, foi possível responder à questão norteadora dessa pesquisa, conforme apresentado na seção 6. Por último, na seção 7 são apresentadas as considerações finais e os trabalhos futuros.

\section{Quantos alunos devem participar de uma sessão de bate-papo educacio- nal?}

A conversação por bate-papo deixou de ser aberta e realizada entre estranhos identificados por apelidos (nickname), como na época do IRC, e se tornou uma conversa contextualizada em comunidades virtuais entre participantes identificados por um perfil no sistema de redes sociais [8]. Uma comunidade virtual pode ser uma turma de uma disciplina, uma equipe de trabalho, um grupo de amigos, ou um agrupamento de pessoas interessadas num tema. Desta forma, além da socialização e recreação, os sistemas de bate-papo passaram a ser usados para dar suporte a diferentes processos de trabalho em grupo: debates, reuniões, entrevistas etc.

O bate-papo é um meio de conversação síncrono em que são trocadas pequenas mensagens textuais publicadas para todos os conectados na mesma sala de bate-papo [9]. Embora a troca de mensagens seja realizada por escrito, os participantes sentem como se estivessem conversando oralmente. Os interlocutores até modificam a linguagem formal escrita para torná-la mais parecida com a conversação face a face, por exemplo, fazendo uso de onomatopeias, alongamentos vocálicos e sobrecarga de pontuação. Esse fenômeno, denominado "re-oralização da língua escrita" [10], indica a ocorrência de características da conversação oral no texto produzido numa sessão de bate-papo.

$\mathrm{Na}$ educação, o bate-papo possibilita a constituição de um espaço para realizar atividades educacionais diferentes da aula tradicional. No bate-papo geralmente ocorre intensa troca de mensagens e descaracterização do professor como detentor do conhecimento e da palavra. A conversação informal, típica do bate-papo, possibilita o aluno perceber melhor o outro e perceber-se melhor como parte do grupo, pois proporciona um espaço para emoções que diminuem a sensação de impessoalidade e isolamento. Em cursos a distância, o uso do bate-papo contribui para evitar a evasão mantendo os alunos motivados e engajados na disciplina [11].

Quando o bate-papo é usado para realizar atividades educacionais ou de trabalho em grupo, os participantes precisam acompanhar a conversação e compreender cada mensagem. A necessidade de acompanhar a conversação é menor em bate-papo de socialização e recreação, pois os participantes não estão comprometidos em entender a conversação e até podem deixar de ler algumas mensa- 
gens. Já no bate-papo com propósitos educacionais, há o comprometimento em ler todas as mensagens e entender o que está sendo discutido.

Silva [12] afirma que o sistema de bate-papo é o espaço virtual mais parecido com o da sala de aula tradicional e, por ser síncrono, permite a construção do sentimento de pertencimento. Já Hrastinski [5] defende que o uso de sistemas síncronos em conjunto com sistemas assíncronos contribui para o aumento da participação dos alunos na educação online.

No Brasil, no documento sobre "Referenciais de qualidade para educação superior a distância" [13], são enumeradas oito categorias que todo projeto político pedagógico de um curso superior a distância precisaria contemplar, dentre elas, os "(ii) Sistemas de Comunicação":

$O$ desenvolvimento da educação a distância em todo o mundo está associado à popularização e democratização do acesso às tecnologias de informação e de comunicação. No entanto, o uso inovador da tecnologia aplicada à educação deve estar apoiado em uma filosofia de aprendizagem que proporcione aos estudantes efetiva interação no processo de ensinoaprendizagem (...) Da mesma forma que a interação entre professor-estudante, tutor-estudante e professor-tutor deve ser privilegiada e garantida, a relação entre colegas de curso também necessita de ser fomentada. Principalmente em um curso a distância, esta é uma prática muito valiosa, capaz de contribuir para evitar o isolamento e manter um processo instigante, motivador de aprendizagem, facilitador de interdisciplinaridade e de adoção de atitudes de respeito e de solidariedade ao outro, possibilitando ao estudante o sentimento de pertencimento ao grupo. [Ibidem, p.10]

Ainda no documento sobre os referenciais de qualidade para EAD, dentre outros aspectos relacionados aos sistemas de comunicação, destaca-se: "valer-se de modalidades comunicacionais síncronas como videoconferências, chats (...) para promover a interação em tempo real entre docentes, tutores e estudantes" [Ibidem, p.12].

A grande popularidade e a reconhecida utilidade do bate-papo nas práticas pedagógicas online motivaram a realização da presente pesquisa. Buscou-se equacionar quantos alunos podem participar de uma sessão de batepapo sem decair a participação esperada pelo professor. A participação online de alunos é apresentado na literatura como um fenômeno complexo e sua avaliação envolve aspectos quantitativos e qualitativos. A métrica mais comumente usada para participação de alunos é a quantidade de mensagens trocadas sendo seguida pela qualidade das mensagens [14]. Em estudo sobre a participação online, são propostos níveis de participação em que quanto maior o nível, melhor a participação. O nível de parti- cipação que se deseja garantir com a presente pesquisa é o nível 4 - participação como escrita e leitura de mensagens. Saber quantos alunos no máximo devem participar de uma sessão é importante, por exemplo, para planejar a carga-horária necessária para a realização das atividades pedagógicas mediadas pelo bate-papo. Se o tutor modera a sessão de bate-papo entre os alunos, e sendo este tutor devidamente pago por sua hora de trabalho em cada sessão de bate-papo, estimar se os alunos podem ser agrupados de 100 em 100 ou apenas de 5 em 5 tem um grande impacto no planejamento e no custo das atividades acadêmicas. Por exemplo, numa disciplina com 500 alunos online, dependendo do tamanho do grupo definido para a realização de um debate síncrono, serão necessárias serão necessárias um número específico de sessões, por exemplo, 5 sessões para grupos de 100 alunos, 20 sessões para grupos de 25 alunos ou 50 sessões para grupos de 10 alunos. Quanto maior o grupo de alunos numa sessão de bate-papo, menor o custo dessa atividade. Contudo, quanto mais alunos na sessão, entende-se que menor será o nível de participação no bate-papo.

Stahl [15] chama atenção para o impacto do tamanho do grupo sobre a eficácia dos diferentes meios de comunicação; ele defende que grupos pequenos, de três ou quatro alunos ativos, são mais complexos e interessantes do que uma dupla respondendo um ao outro. Contudo, se o grupo for grande demais ocorrerão problemas. A "confusão no bate-papo" [16] é definida quando os participantes não conseguem acompanhar a discussão pela ocorrência de fenômenos, como por exemplo, a sobrecarga de mensagens causada pela grande quantidade de mensagens postadas em um curto intervalo de tempo decorrente de um número excessivo de participantes na sessão de batepapo. Alguns autores, a partir de dados empíricos e em contextos específicos, indicam que o número ideal de participantes num bate-papo é entre 4 e 5 pessoas $[3,17$, 18]; outros estudos defendem que o bate-papo é mais produtivo com 7 ou mais participantes [6]; para Hrastinski [19] o grupo deve ser inferior a 10 participantes; outros estudiosos afirmam que 10 é um tamanho bom para o grupo de participantes [20]; e existe ainda, estudo quantificando de 6 a 18 alunos [11]. Alguns sistemas de batepapo limitam o número de participantes a 30 usuários por sessão, e há sistemas que não estabelecem limites. Com essa diversidade de sugestões não convergentes da quantidade de alunos que devem participar de uma sessão de bate-papo educacional, foi identificada a necessidade de equacionar essa questão - objetivo dessa pesquisa.

Deve existir um limite máximo de participantes a partir do qual ocorre confusão demais e compromete o nível de participação na sessão de bate-papo. Num bate-papo educacional é importante que todos consigam ler todas as mensagens e que também possam enviar mensagens de vez em quando. Essas restrições não acontecem num 
bate-papo recreativo (para socialização ou namoro) em que o participante não tem a necessidade de acompanhar a discussão nem se vê obrigado a escrever mensagens, não precisa ler tudo e pode manter-se calado. Numa sessão recreativa não há um fator limitante para a quantidade de pessoas no bate-papo. Só faz sentido discutir a quantidade máxima de participantes se for exigido um nível mínimo de participação, pois caso a participação possa ser zero (o aluno não precisa ler nem escrever mensagens), tal como ocorre na transmissão de um vídeo em broadcasting, então não há um limite máximo de alunos: o professor pode dar uma aula por bate-papo para milhares de alunos conectados ao mesmo tempo desde que somente o professor esteja digitando as mensagens e a quantidade de mensagens produzidas por aluno tenda a zero. O problema surge quando se dá a chance para o aluno interagir com o professor e com os colegas. Desta forma, é desejável determinar a quantidade máxima de participantes numa sessão de bate-papo em que seja possível alcançar uma participação definida em termos da quantidade de mensagens lidas e produzidas por participante. Considerando que todos devem ler todas as mensagens, então o nível de participação irá variar em função da quantidade das mensagens produzidas.

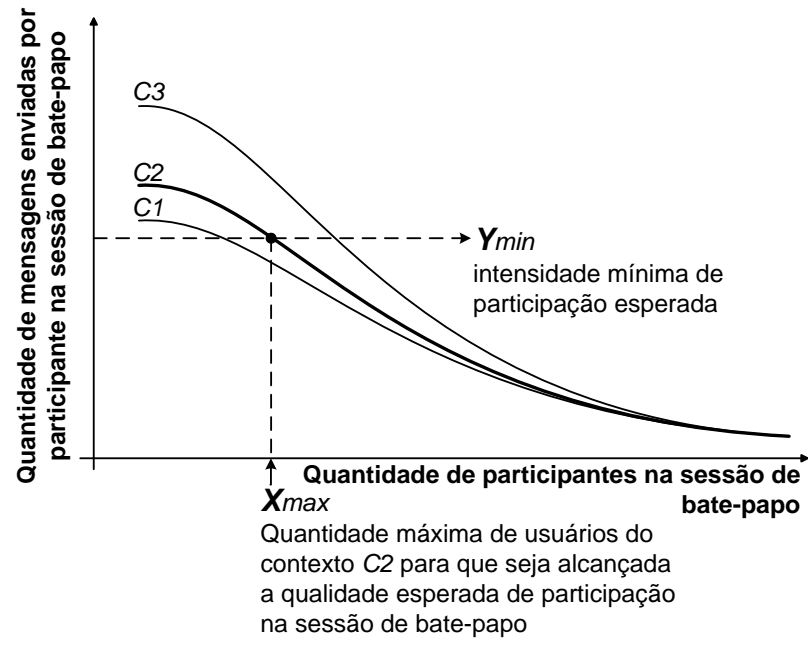

Figura 1: Modelo idealizado da participação em bate-papo educacional

Conforme aumenta a quantidade de participantes na sessão, mais mensagens são produzidas, o que demanda de cada participante mais tempo para ler todas as mensagens e assim cada indivíduo tem menos tempo para enviar novas mensagens. Embora a produção total de mensagens aumente com a quantidade de participantes, também é fato que a produção individual de mensagens decai com o aumento do número de participantes. Na Figura 1 é ilustrado um modelo idealizado da produção de mensagens por pessoa em função da quantidade de participantes na sessão de bate-papo.

No modelo da Figura 1 são apresentadas curvas dife- rentes - $\mathrm{C} 1, \mathrm{C} 2$ e $\mathrm{C} 3$ - que representam contextos distintos (turmas diferentes). Os contextos representam as características singulares de cada grupo tais como: idade média, nível de instrução, assunto debatido, protocolo da conversação etc. Na presente pesquisa, deseja-se determinar, para um dado contexto, qual a quantidade máxima de participantes $\left(X_{\max }\right)$ que deve estar na sessão para que seja possível alcançar uma determinada produção média de mensagens $\left(Y_{\min }\right)$.

\section{Ações realizadas numa sessão de ba- te-papo educacional}

Durante uma sessão de bate-papo, o participante lê e escreve mensagens, e também reflete sobre a discussão. Essas ações, descritas nas subseções a seguir, são os comportamentos elementares de qualquer participante de um bate-papo educacional e constituem a base do modelo matemático apresentado na seção seguinte.

\subsection{Leitura}

Uma pessoa não lê igual à outra pessoa; a velocidade de leitura depende do nível de escolaridade, da experiência em bater-papo, do grau de interesse e de conhecimento sobre o assunto em discussão, dentre outras características do indivíduo. O tempo de leitura também depende de fatores externos ao sujeito, como o tamanho da mensagem e a interface do sistema de bate-papo.

Para caracterizar a ação de leitura de mensagens de bate-papo, foram investigados quais fatores externos influenciam o processo de leitura. Foi realizado um experimento com 6 alunos de pós-graduação em Informática, sendo 4 homens e 2 mulheres com idade entre 24 e 55 anos. Para medir o tempo de leitura, cada indivíduo leu mensagem-a-mensagem de um $\log$ real de bate-papo educacional pela tela do computador. Quando o indivíduo pressionava uma tecla, uma nova mensagem era apresentada e era registrado o tempo de leitura decorrido entre uma tecla e a seguinte. Cada participante do experimento leu um total de 110 mensagens, sendo descartado o tempo de leitura das 10 primeiras mensagens. Buscou-se caracterizar como ocorre a leitura de mensagens entre diferentes indivíduos ao ler mensagens de diferentes tamanhos, em logs sobre diferentes assuntos e apresentados em diferentes interfaces de bate-papo. Foram testadas as 4 hipóteses a seguir:

(i) Os alunos leem com velocidades significativamente diferentes.

(ii) $\mathrm{O}$ tempo de leitura varia em função da quantidade de caracteres na mensagem;

(iii) $\mathrm{O}$ tempo de leitura varia em função do assunto em discussão; 
(iv) A interface do sistema de bate-papo influencia o tempo de leitura das mensagens.

Para testar estas hipóteses, cada aluno deveria ler 3 logs reais de bate-papo, sendo: dois logs sobre um mesmo assunto e um terceiro sobre assunto diferente; e dois desses logs foram apresentados num bate-papo típico e um deles foi apresentado numa interface de bate-papo com avatar. Após coletado o tempo de leitura de cada participante de cada mensagem dos três logs, por meio de testes estatísticos, foi possível concluir quais dos fatores levantados de fato influenciam o tempo de leitura. Todas as análises foram realizadas com o uso do software estatístico $\mathrm{R}[21]$ e o nível de significância adotado foi o de $5 \%$. Com a intenção de verificar qual melhor conjunto de testes a ser usado (paramétricos ou não paramétricos), testou-se inicialmente se os tempos de leitura seguiam a distribuição normal por meio do teste Shapiro-Wilk. Para esse teste, foi obtido um valor $\mathrm{p}<0,05$, o que rejeita a hipótese de os dados seguirem a distribuição normal. A partir desse resultado, o teste não paramétrico de Kruskal-Wallis foi usado para testar as hipóteses, para isso, foi verificado se havia diferença significativa entre os tempos de leitura obtidos entre os indivíduos em: todos os três $\log s$; entre os $\log s 1$ e 2 (se a interface do sistema influencia); e entre os $\log s 1$ e 3 (se o assunto influencia).

Para inferir sobre a velocidade de leitura dos indivíduos (hipótese i), foi verificado se há diferença significativa na distribuição dos tempos que cada um dispendeu ao ler as mensagens do $\log 1$. Os resultados obtidos pela aplicação do teste foi um p-valor $<0,001$, o que possibilitou concluir que os sujeitos realmente leem de forma significativamente diferente, com diferentes velocidades.

Para testar a hipótese (ii), que o tempo de leitura é proporcional à quantidade de caracteres da mensagem, foi verificada a correlação entre a quantidade de caracteres das mensagens de cada $\log$ e os tempos de leitura de cada participante. A média das correlações foi de 0,72 , o que, de acordo com Shimakura [22], possibilita interpretar que a correlação entre essas variáveis é forte, possibilitando concluir que o tempo de leitura é realmente proporcional à quantidade de caracteres (ainda que cada sujeito tenha uma velocidade distinta de leitura). A correlação entre o tempo de leitura e o tamanho da mensagem é ilustrada para um dos participantes do estudo na Figura 2.

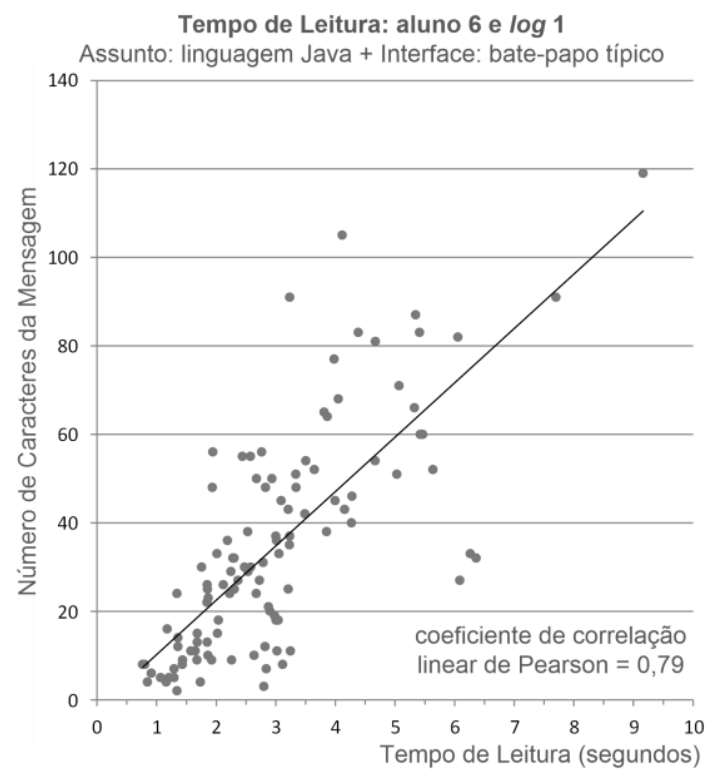

Figura 2: Correlação entre o tempo de leitura e o tamanho da mensagem de um dos participantes

Sobre a influência do assunto (hipótese iii), o teste comparando os tempos de leitura dos logs 1 e 3 (em que foram abordados assuntos diferentes) não apontou diferença significativa com $p$-valor $>0,05$. Ao menos a partir desse experimento não foi possível concluir que assuntos distintos provocam diferenças significativas na velocidade de leitura dos indivíduos.

Sobre a hipótese (iv), concluiu-se que os sujeitos leem em tempos significativamente diferentes dependendo da interface do sistema de bate-papo.

Dado que a velocidade de leitura é uma característica individual e que depende da quantidade de caracteres a serem lidos, e considerando que não há diferença significativa na velocidade de leitura em função do assunto em discussão e que a interface do sistema é a mesma durante toda uma sessão de bate-papo - a partir desses resultados foi assumido, por simplificação, que o tempo de leitura $\bar{T}_{L}$ pode ser modelado por uma equação linear em função da quantidade de caracteres da mensagem.

$\bar{T}_{L}=1+(0,04 *$ média de caracteres por mensagem $)$

A equação (1) representa o tempo médio, em segundos, que os sujeitos do experimento dispenderam para a leitura das mensagens daquelas sessões de bate-papo (calculada a partir das linhas de tendência linear da correlação entre o tempo de leitura e a quantidade de caracteres por mensagem por indivíduo). É preciso ressaltar que essa equação é válida para o grupo de alunos do experimento e que para outro perfil de alunos os coeficientes dessa equação são diferentes. 


\subsection{Escrita}

Cada pessoa possui uma velocidade de digitação específica. Não só a velocidade de digitação, mas também os padrões de digitação diferem de um indivíduo para outro. O intervalo entre o pressionar de uma tecla e a outra, e o tempo em que uma tecla permanece pressionada, são características únicas de cada indivíduo e corroboram para o estabelecimento de um padrão de digitação individual $[23,24]$. Esses padrões são estudados na área de Biometria da Digitação.

Para o presente artigo, é de interesse obter tempos médios de digitação de mensagens durante a participação em bate-papos educacionais, para se caracterizar essa atividade e posteriormente parametrizar o modelo de participação. Faz-se então necessário medir a velocidade de elaboração de mensagem dos participantes. Para efetivar essa medição, foi desenvolvido um mecanismo que captura o tempo de cada tecla pressionada durante a digitação de uma mensagem de bate-papo. Foi realizado um estudo, com profissionais de computação, em que os sujeitos digitaram algumas mensagens e o tempo de digitação foi registrado e posteriormente analisados. Nesse experimento, o tamanho médio das mensagens digitadas foi de 85 caracteres por mensagem e o tempo médio de digitação foi de 24 segundos por mensagem, resultando na velocidade média de digitação de 1 caractere / 0,28 segundos. Verificou-se uma correlação muito forte $(0,95)$ entre a quantidade de caracteres e o tempo de digitação da mensagem. A partir desse resultado foi assumido que o tempo de escrita $\bar{T}_{E}$ pode ser modelado por uma equação linear em função da quantidade de caracteres da mensagem.

$$
\bar{T}_{E}=0,28 * \text { média de caracteres por mensagem }
$$

A equação (2) descreve o tempo médio de escrita de mensagens, medido em segundos, dispendido pelos sujeitos do grupo do experimento.

\subsection{Reflexão}

Os participantes não ficam lendo e digitando o tempo todo durante a sessão de bate-papo. Ocorrem intervalos, por exemplo entre o término da leitura das mensagens publicadas até aquele momento e a chegada de uma nova mensagem a ser lida, em que o participante não está digitando nem lendo mensagens. Nestes momentos o usuário encontra-se refletindo sobre a discussão.

O tempo de reflexão é o tempo que sobra de uma sessão de bate-papo desconsiderando o tempo em que o usuário fica escrevendo suas mensagens e o tempo em que fica lendo as mensagens. Assim, nesta pesquisa, o tempo de reflexão foi estimado por meio da equação (3).

$T_{R}=$ duração da sessão $-\left(\bar{T}_{L} * \bar{m} * p\right)-\left(\bar{T}_{E} * \bar{m}\right)$ onde $T_{R}$ é o tempo total de reflexão numa sessão de batepapo, $\bar{m}$ é a quantidade média de mensagens enviadas por participante na sessão, e $p$ é a quantidade de participantes na sessão.

\section{Modelagem da participação em ba- te-papo educacional}

Para modelar a dinâmica de participação no bate-papo educacional foi utilizada a Teoria de Filas [7] em que cada participante foi representado como um servidor que precisa ler (atender) todas as mensagens (clientes) digitadas pelos participantes da sessão e enfileiradas na ordem em que são enviadas (publicadas na sala de bate-papo) Figura 3.

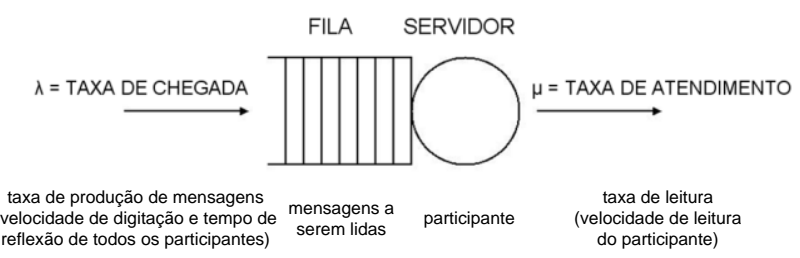

Figura 3: Teoria de filas para modelar a participação

Cada participante, além de ser um servidor, também desempenha o papel de uma fonte geradora de mensagens enviadas para a fila de todos os participantes da sessão. A taxa de leitura de mensagens $\mu$ é o inverso do tempo médio de leitura $\bar{T}_{L}$, e a taxa de produção de mensagens $\lambda$ é o que se deseja determinar dado o tamanho $p$ do grupo de participantes. Os tempos de leitura e de produção de mensagens foram considerados, na presente pesquisa, como exponencialmente distribuídos.

Para obter as estimativas de produção de mensagem a partir dessa modelagem, o modelo foi implementado no Tangram-II [25], que é um ambiente de modelagem baseado no paradigma orientado a objetos e que fornece uma variedade de solvers para obtenção das medidas de interesse. As estimativas produzidas a partir do modelo elaborado na presente pesquisa, discutidas nas próximas seções, foram obtidas a partir da Solução Analítica do Tangram-II.

\subsection{Modelagem do comportamento de um usuário de bate-papo}

Para modelar o comportamento de um participante da sessão de bate-papo educacional, foi desenvolvido um diagrama de estados para representar as atividades elementares que o participante realiza durante o bate-papo: ler e escrever mensagens - Figura 4. Nessa abordagem, o participante permanece no estado Escrevendo por um determinado tempo até que envia a mensagem aos demais participantes. Após enviar a mensagem, ele verifica se existem mensagens a serem lidas, e se houver passa para o estado Lendo. Enquanto o participante está Escrevendo 
ou Lendo mensagens, outras mensagens podem chegar e essas são enfileiradas na ordem em que são enviadas pelos demais participantes em um Fila de Mensagens. A transição de estados é modelada em função de taxas. $\mathrm{O}$ participante permanece Lendo uma determinada mensagem por um tempo médio de leitura $\left(\bar{T}_{L}\right)$, e a taxaLeitura é calculada como o inverso do tempo médio. Da mesma forma, o participante permanece no estado Escrevendo durante um tempo médio de escrita $\left(\bar{T}_{E}\right)$, a partir do qual se calcula a taxa de escrita.

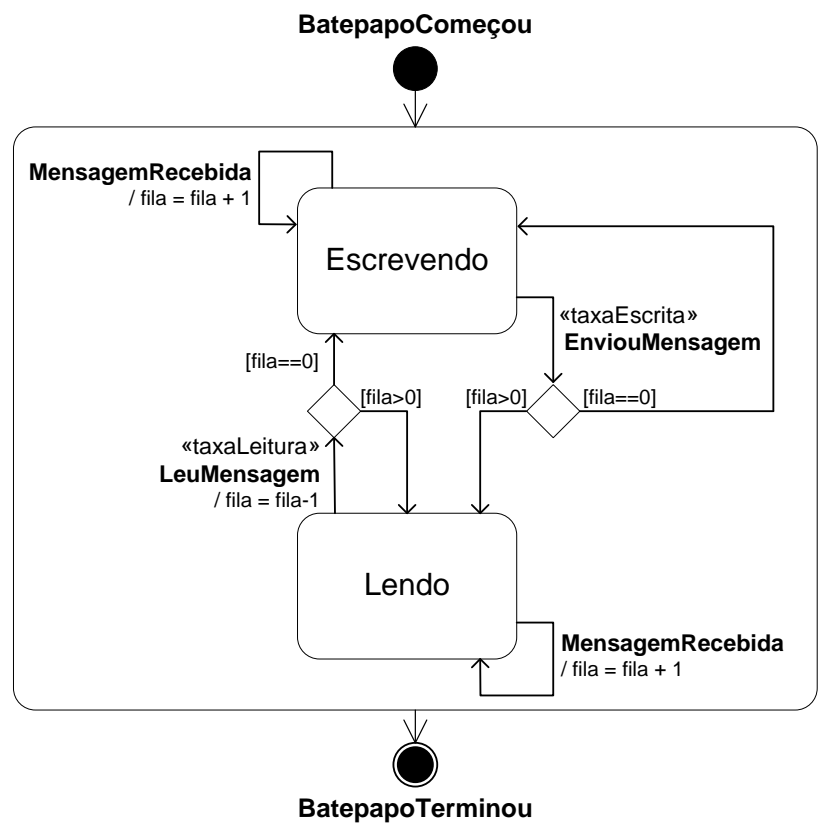

Figura 4: Diagrama dos Estados Lendo e Escrevendo

Representar um participante de bate-papo por apenas dois estados é irreal, pois pressupõe que o participante fica o tempo todo ou Lendo ou Escrevendo mensagens. Contudo, essa simplificação possibilita estimar um Limite Máximo de mensagens produzidas pelo participante numa sessão de bate-papo pressupondo que esse sujeito se encontra o tempo todo ocupado lendo todas as mensagens publicadas e produzindo o máximo de mensagens que seria capaz. Esse limite máximo de mensagens por indivíduo é calculado pela equação (4).

$$
\begin{aligned}
& m * \bar{T}_{E}+m * p * \bar{T}_{L}=T_{\text {sessão }} \\
& m=T_{\text {sessão }} /\left(\bar{T}_{E}+p * \bar{T}_{L}\right)
\end{aligned}
$$

onde $m$ representa o número de mensagens produzidas por participante em uma sessão de 60 minutos.

O Limite Máximo de produção de mensagens corrobora para a avaliação do modelo de participação, como será discutido na Seção 5.

O diagrama de estados apresentado na Figura 5 é mais próximo do comportamento real dos usuários, pois leva em conta que um participante, além de ler e escrever mensagens, também reflete em alguns momentos. Nesse diagrama, o indivíduo vai para o estado Refletindo quando não tem mais mensagens a serem lidas, e fica refletindo por um tempo até que: chega uma nova mensagem a ser lida e, imediatamente, o usuário volta para o estado Lendo; ou fica refletindo por algum tempo até que decide escrever uma nova mensagem, indo para o estado Escrevendo.

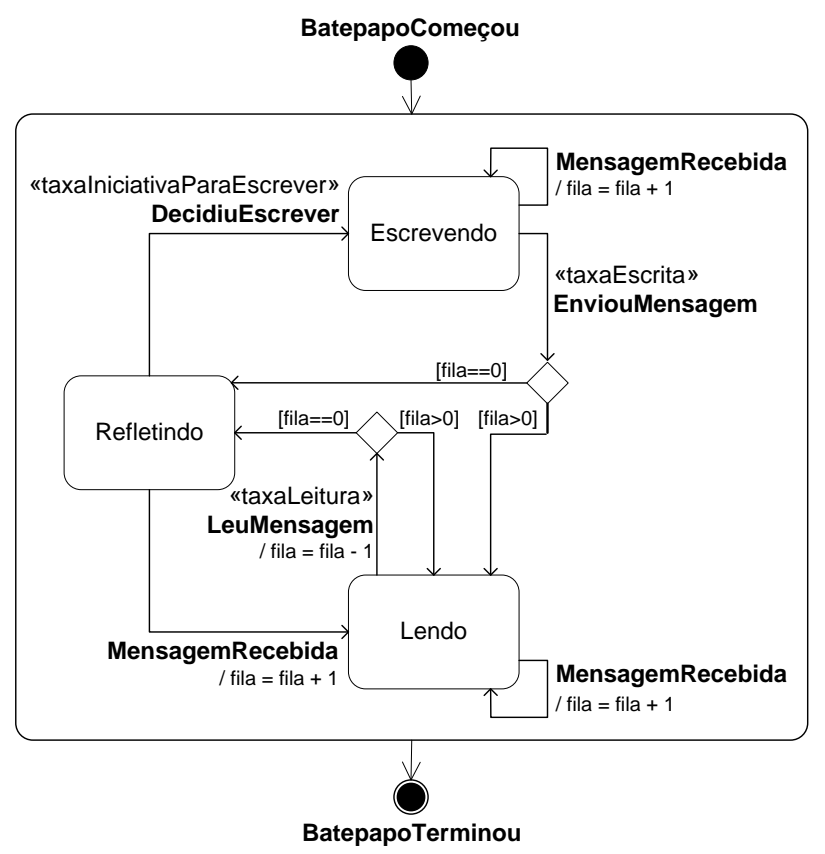

Figura 5: Diagrama dos Estados Lendo, Escrevendo e Refletindo

Como discutido na Seção 5, esse diagrama com três estados foi implementado no modelo e mostrou-se adequado, pois foram produzidas estimativas compatíveis com os dados das sessões reais de bate-papo educacional.

\subsection{Modelagem da interação entre os partici- pantes da sessão de bate-papo}

Numa primeira estratégia de modelagem para a implementação do modelo no Tangram-II, foi definido um objeto para representar cada participante individualmente, conforme ilustrado na Figura 6. Cada objeto Participante realiza as atividades apresentadas no diagrama da Figura 5: lê e escreve mensagens, e fica refletindo. Cada Participante também é caracterizado por suas taxas de leitura, escrita e iniciativa para escrever mensagens. Os participantes enviam mensagens uns para os outros e também leem as mensagens enviadas de acordo com suas respectivas taxas. As mensagens recebidas são enfileiradas para a posterior leitura caso o participante esteja ocupado naquele momento lendo outras mensagens ou escrevendo uma nova mensagem. 


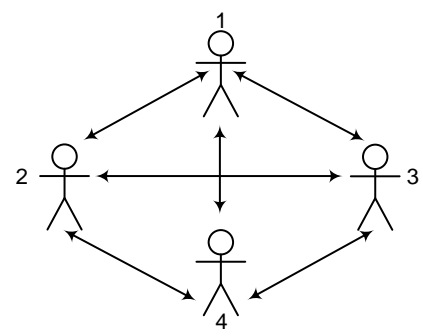

Figura 6: Estratégia de modelagem: Interação entre Indivíduos

Nessa estratégia de modelagem, cada Participante é representado por duas variáveis: Fila, que contém a quantidade de mensagens a serem lidas; e Estado, que representa a atividade realizada pelo Participante em um determinado momento da sessão de bate-papo. O conjunto de todos os estados dos $p$ participantes, bem como as transições possíveis entre os estados, são representados por uma Cadeia de Markov [26].

Seguindo o paradigma do Tangram-II, o objeto Participante é replicado $n$ vezes, um para cada participante do grupo da sessão. Essa estratégia de modelagem, contudo, provoca uma explosão de estados da cadeia de Markov, o que impossibilita obter a medida de interesse pelo Tangram-II para grupos com 6 ou mais participantes.

Para contornar a explosão de estados, uma segunda estratégia de modelagem foi elaborada - Figura 7. Nessa nova abordagem, aqui denominada Interação IndivíduoGrupo, um único objeto Participante interage com uma entidade única que representa os demais participantes do grupo produzindo mensagens.

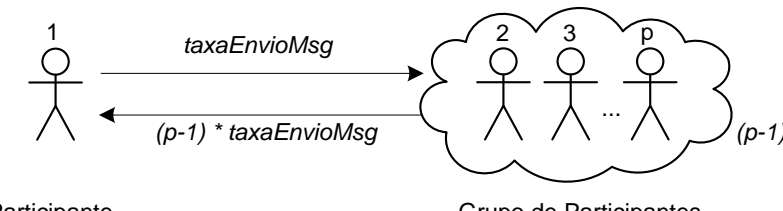

Figura 7: Estratégia de modelagem: Interação Indivíduo-Grupo

Enquanto na estratégia Interação entre Indivíduos ocorre o aumento exponencial do número de estados conforme aumenta a quantidade de participantes da sessão de bate-papo, na estratégia Interação IndivíduoGrupo apenas dois objetos interagem, não variando em função da quantidade de integrantes do grupo, e consequentemente não provocando a explosão dos estados, o que possibilitou obter pelo Tangram-II as estimativas de interesse dessa pesquisa.

Nessa estratégia, cada participante do Grupo deve produzir mensagens numa taxa semelhante ao do indivíduo isolado, pois assume-se que todos os participantes do bate-papo tem um comportamento similar. Isto significa que a taxa de envio do Grupo dividida por $(p-1)$ precisa ser próxima ao número de mensagens enviadas pelo indivíduo isolado. $\mathrm{O}$ valor da taxa de mensagens enviadas pelo indivíduo isolado é a medida de interesse que se quer encontrar. Para obter essa taxa, o primeiro passo é atribuir um valor aleatório para a taxa de produção de mensagens pelo grupo e, pelo Tangram-II, calcular a taxa de envio de mensagens do indivíduo isolado. Em seguida compara-se a taxa atribuída ao sujeito do grupo e a produzida pelo indivíduo isolado. A diferença entre as taxas deve ser a menor possível - nessa pesquisa, foi definido que a diferença deveria ser inferior a 1 mensagem. Em função do resultado obtido pelo Tangram-II, ajusta-se a estimativa da taxa de envio de mensagens do grupo até que a taxa dos indivíduos do grupo fique próxima da taxa do indivíduo isolado. Desta forma, iterativamente, obtémse uma estimativa para a quantidade de mensagens produzidas por participante dado um determinado tamanho do grupo - essa é a medida de interesse dessa pesquisa. Como exemplo, na Tabela 1 são exemplificados os passos realizados iterativamente para se obter a estimativa da produção individual de mensagens considerando que há 7 participantes na sessão (um indivíduo mais 6 integrantes no grupo).

\begin{tabular}{|c|c|}
\hline $\begin{array}{l}\text { "Chute" inicial: Taxa de } \\
\text { envio de mensagens do Grupo } \\
\text { (e a respectiva quantidade } \\
\text { de mensagens enviadas por } \\
\text { cada participante do Grupo) }\end{array}$ & $\begin{array}{l}\text { Valor calculado } \\
\text { pelo Tangram-II: } \\
\text { quantidade de } \\
\text { mensagens envia- } \\
\text { das pelo Parti- }\end{array}$ \\
\hline $\begin{array}{c}\text { Mensagens por indivíduo do } \\
\text { grupo durante } 1 \mathrm{~h} \text { de sessão } \\
=\text { taxa } * 60 \mathrm{~min} / 6 \text { partici- } \\
\text { pantes }\end{array}$ & $\begin{array}{c}\text { cipante Isolado } \\
\text { durante } 1 \mathrm{~h} \text { de } \\
\text { bate-papo }\end{array}$ \\
\hline $3,3 \quad(33,0)$ & 34,4 \\
\hline $3,4 \quad(34,0)$ & 34,2 \\
\hline $3,5 \quad(35,0)$ & 34,0 \\
\hline
\end{tabular}

Tabela 1: Obtenção da estimativa de mensagens enviadas adotando a estratégia de Interação Indivíduo-Grupo (sessão de 60 minutos e 7 participantes)

Conforme apresentado na Tabela 1, foram testadas sucessivas taxas de envio de mensagens do Grupo. Dentre as taxas testadas, a que mais aproximou a quantidade de mensagens enviadas por cada participante do grupo com a do participante isolado foi 3,4. Para essa taxa, cada participante do grupo teria produzido 34,0 mensagens, enquanto o Participante Isolado, conforme o cálculo produzido pelo Trangram-II, teria enviado 34,2 mensagens durante 60 minutos. Essa diferença é inferior a 1 mensagem, e restringindo a precisão da taxa a uma casa decimal, essa é a taxa que mais se aproxima da produção do indivíduo isolado com a do indivíduo do grupo. Repetindo esse trabalho para diferentes quantidades de participantes no grupo, obtém-se a curva de interesse: quantidade de mensagens produzidas por um participante da sessão de bate-papo em função dos diferentes tamanhos de grupo. 


\section{Avaliação do Modelo}

Para avaliar a adequação do modelo elaborado nesta pesquisa, as estimativas produzidas pelo modelo foram comparadas com os dados reais obtidos de um corpus de análise. O corpus de análise utilizado é constituído de logs de sessões de bate-papo das turmas de uma disciplina lecionada totalmente a distância, denominada "Tecnologias de Informação Aplicadas à Educação" (TIAE) [27]. Ao todo foram obtidos 142 logs de bate-papo ocorridos em 18 turmas ministradas entre os anos de 1999 e 2008 (em cada turma foram realizadas, em média, 8 sessões de $1 \mathrm{~h}$ de bate-papo).

\subsection{Estimativa dos parâmetros do modelo para as turmas TIAE}

Os parâmetros do modelo são: taxaLeitura, taxaEscrita e taxaIniciativaParaEscrever - esses parâmetros indicam a frequência de ocorrência de cada um dos eventos que compõem os estados do diagrama ilustrado na Figura 5. Os parâmetros encapsulam os contextos em que o modelo é aplicado. Os contextos representam as características singulares de cada grupo tais como: idade média, nível de instrução, assunto debatido, protocolo da conversação etc. Além dessas taxas, também é preciso especificar a função de distribuição de probabilidade de cada evento.

Uma vez que não é possível voltar no tempo para medir a velocidade de leitura e de digitação dos participantes das sessões de bate-papo que compõem o corpus de análise, então optou-se por estimar os parâmetros de cada turma TIAE com base nas equações apresentadas na seção 3. Considerou-se que o tempo médio de leitura da equação (1) é uma aproximação razoável para o contexto das turmas TIAE, pois ambos contextos são compostos de alunos de graduação e pós-graduação em Informática. Para estimar o parâmetro taxaLeitura das turmas TIAE, além da velocidade de digitação também é preciso considerar o tamanho médio das mensagens emitidas em cada turma, sendo este um parâmetro obtido dos logs. Na equação (5) é exemplificada a aplicação da equação (1) para estimar o parâmetro taxaLeitura dos participantes da turma TIAE2007.1 (primeiro semestre de 2007), onde:

$$
\begin{aligned}
\bar{T}_{L} & =1+(0,04 * \text { média de caracteres }) \\
& =1+(0,04 * 94,1)=1+3,76=4,76 \mathrm{seg}=0,08 \mathrm{~min}
\end{aligned}
$$$$
\text { taxaLeitura }=1 / 4,76=0,21 \mathrm{msg} / \mathrm{seg}=12,6 \mathrm{msg} / \mathrm{min}
$$

Para estimar as taxas de escrita dos participantes das turmas TIAE, na impossibilidade de se obter esse parâmetro a partir do $\log$, considerou-se que a equação (2) é uma aproximação razoável. Esta equação foi parametrizada com o tamanho médio das mensagens de cada turma TIAE (obtido dos logs reais). Na equação (6), é exempli- ficada a aplicação da equação (2) para estimar o parâmetro taxaEscrita dos participantes da turma TIAE2007.1, onde:

$$
\begin{aligned}
& \begin{aligned}
\bar{T}_{E} & =0,28 * \text { média de caracteres por mensagem } \\
& =0,28 * 94,1=26,3 \mathrm{seg}=0,44 \mathrm{~min}
\end{aligned} \\
& \text { taxaEscrita }=1 / 0,44=2,3 \mathrm{msg} / \mathrm{min}
\end{aligned}
$$

Para estimar as taxaIniciativaParaEscrever dos participantes das turmas TIAE, foi primeiramente utilizada a equação (3) para estimar o tempo de reflexão total na sessão de bate-papo $\left(T_{R}\right)$ considerando: duração da sessão (obtida de cada $\log$ ), a quantidade de participantes de cada turma TIAE; a média de mensagens enviadas por participante; e os tempos de leitura e de escrita estimados de cada turma. Considerando-se que o participante envia em média $\bar{m}$ mensagens durante a sessão de bate-papo, então entre a escrita de uma mensagem e a outra esse participante fica refletindo, em média, $T_{R} / \bar{m}$. Optou-se em adotar esse valor como uma aproximação do tempo médio de iniciativa de escrita de mensagens $\left(\bar{T}_{I E}\right)$. A taxa de iniciativa para escrever é então obtida pelo inverso de $\bar{T}_{I E}$. O procedimento elaborado para a estimativa desses parâmetros na turma TIAE2007.1 é exemplificado na equação (7).

$T_{R}=$ duração da sessão $-\left(\bar{T}_{L} * \bar{m} * p\right)-\left(\bar{T}_{E} * \bar{m}\right)$

$T_{R}=60-(0,08 * 32,8 * 7,9)-(0,44 * 32,8)=24,8 \mathrm{~min}$

$\bar{T}_{I E}=T_{R} / \bar{m}$

$\bar{T}_{I E}=24,8 / 32,8=0,76$

taxaIniciativaParaEscrever $=1 / \bar{T}_{I E}=1 / 0,76=1,3$

Além das taxas, é preciso especificar a função de distribuição de probabilidade dos eventos. Na presente pesquisa, optou-se por utilizar o modelo Poisson com a distribuição exponencial para representar a distribuição de todos os eventos do modelo já que essa distribuição representa um grande número de processos na natureza e é a distribuição mais comumente utilizada em processos modelados como fila. Além disso, de acordo com Fogliatti e Mattos [26], "sempre que possivel, deve ser escolhida a distribuição exponencial mesmo que não seja o melhor ajuste, pois a sua utilização em muito facilita a obtenção das medidas de desempenho do sistema" (p.219).

Com as taxas usadas como parâmetros do modelo considerando os dados de cada turma TIAE, e especificadas as funções de distribuição de probabilidade dos eventos, o modelo pôde ser rodado no ambiente Tangram-II para obter o número de mensagens enviadas em função das seguintes quantidades de participantes: $5,9,12,20$, 30, 50 e 100 participantes. Com os valores calculados pelo modelo e interpolando os valores não calculados, é traçada a "curva de participação" estimada para cada 
turma TIAE. Como exemplo, na Figura 8 é apresentada a curva traçada a partir dos resultados produzidos pelo modelo para a turma TIAE 2007.1.

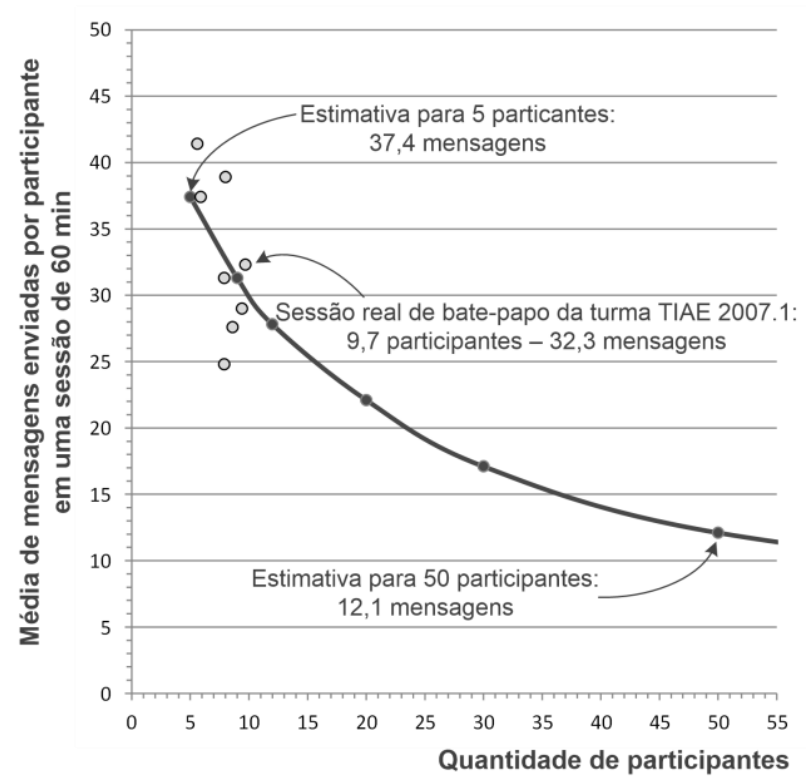

Figura 8: Quantidade de mensagens ocorridas nas sessões TIAE e a curva de participação

Aplicando-se os procedimentos aqui descritos, foi estimada uma curva de participação para cada turma TIAE. Essa estimativa foi utilizada para avaliar a precisão do modelo, como descrito a seguir.

\subsection{Teorema Central do Limite para avaliar a precisão do modelo}

Para avaliar o modelo elaborado, as estimativas produzidas pelo modelo foram comparadas com os dados obtidos dos logs reais. Diferentemente do modelo que estima a produção média de mensagens para cada tamanho do grupo, nos dados reais ocorrem diferentes valores para um mesmo tamanho de grupo - por exemplo, numa turma com 10 participantes, em média cada um produziu 32 mensagens durante uma sessão, mas na sessão seguinte produziram em média 29 mensagens (conforme os dados extraídos da turma TIAE 2007.1 nas sessões 3 e 4). Portanto, se pudéssemos realizar várias sessões com exatamente 10 participantes no grupo, seria possível estimar um valor médio de mensagens produzidas e um intervalo ao redor dessa média que abrigaria a maioria das ocorrências reais.

A precisão da estimativa do modelo em relação aos dados reais é fundamentada pelo Teorema Central do Limite. Pelo Teorema, conforme vai aumentando o tamanho da amostra (ocorrências reais), a distribuição amostral da média aproxima-se de uma distribuição Normal, e neste caso $95 \%$ dos dados amostrais ficam entre $\mu-2 \sigma$ e $\mu+2 \sigma$, onde $\mu$ é a média populacional e $\sigma$ é o desvio pa- drão populacional. Optou-se por um intervalo de confiança de $95 \%$, por ser o tradicionalmente adotado em pesquisa quantitativa [28]. Para usar o intervalo de confiança de $95 \%$, usa-se a área entre $\mu-2 \sigma$ e $\mu+2 \sigma$. Isso implica em verificar se $95 \%$ dos dados reais ocorridos nas sessões da turma TIAE ficam dentro desses limites ao redor da média estimada pelo modelo.

Cada ponto da curva de participação obtida do modelo corresponde à estimativa da média de mensagens enviadas numa amostra $(\bar{x})$ em função do tamanho do grupo de participantes. Falta definir o desvio padrão dessa média de mensagens produzidas na amostra $(s)$. Foram realizados testes estatísticos que possibilitaram concluir que o processo real de produção de mensagens nas turmas TIAE pode ser aproximado por uma Poisson. Assumindo essa distribuição amostral, o desvio padrão é calculado como sendo $\sigma=\sqrt{\lambda}$ (na distribuição de Poisson, a média $\mu$ é representada pela letra grega $\lambda$ [lambda]).

A partir dessa abordagem, foi verificado se $95 \%$ dos dados reais ficavam dentro dos limites calculados em função das médias estimadas pelo modelo. Foram contabilizadas quantas sessões das turmas TIAE ficaram dentro da região estimada em função do modelo e quantos ficaram fora, como exemplifica o procedimento ilustrado Figura 9.

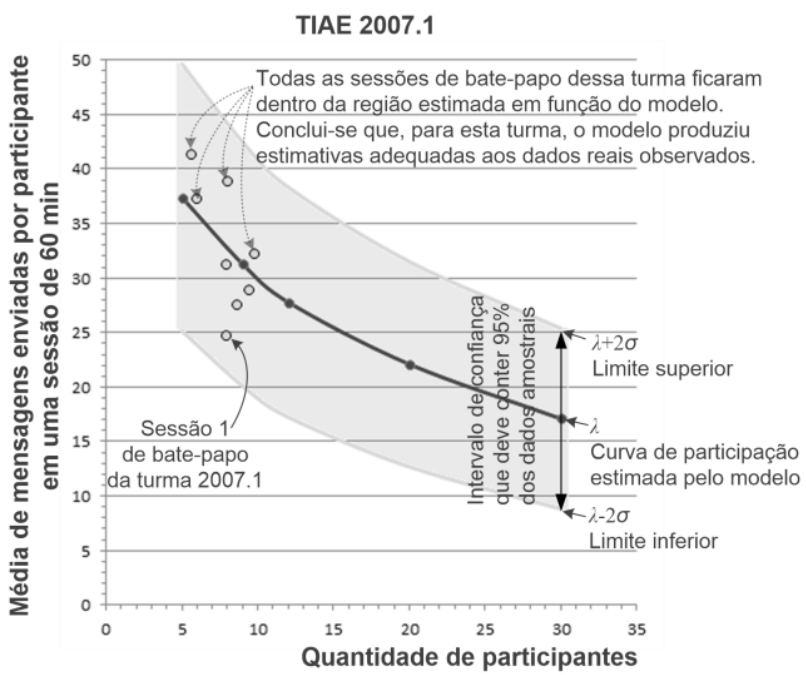

Figura 9: Dados das sessões de bate-papo da turma TIAE $2007.1 \mathrm{em}$ relação à região estimada em função do modelo

De todas as 142 sessões de bate-papo das turmas TIAE, 95,8\% ficaram dentro da região estimada em função do modelo. Este valor está dentro dos limites definidos pelo Teorema Central do Limite $(95,4 \%)$, o que possibilita concluir que o modelo de participação produz estimativas adequadas da média de mensagens enviadas por participante em função do tamanho do grupo presente na sessão de bate-papo educacional. 


\section{Quantidade de Mensagens em Fun- ção da Quantidade de Participantes}

Uma vez que o modelo foi considerado válido, conforme a avaliação apresentada na seção anterior, foi possível buscar uma resposta para a pergunta inicial desta pesquisa: quantos alunos devem participar de uma sessão de bate-papo educacional?

A primeira constatação sobre essa questão é que não existe uma resposta única, pois o limite de alunos depende do contexto de cada turma: do perfil dos alunos (velocidade de leitura e de digitação) e das características da sessão de bate-papo (interface do sistema, protocolo de conversação etc.). No modelo, essas características são encapsuladas nos parâmetros Tempo Médio de Escrita, Leitura e Reflexão. Estimados esses parâmetros para uma turma, ou para o perfil médio de alunos de um curso, o modelo é usado para traçar a curva de participação como a ilustrada na Figura 10.

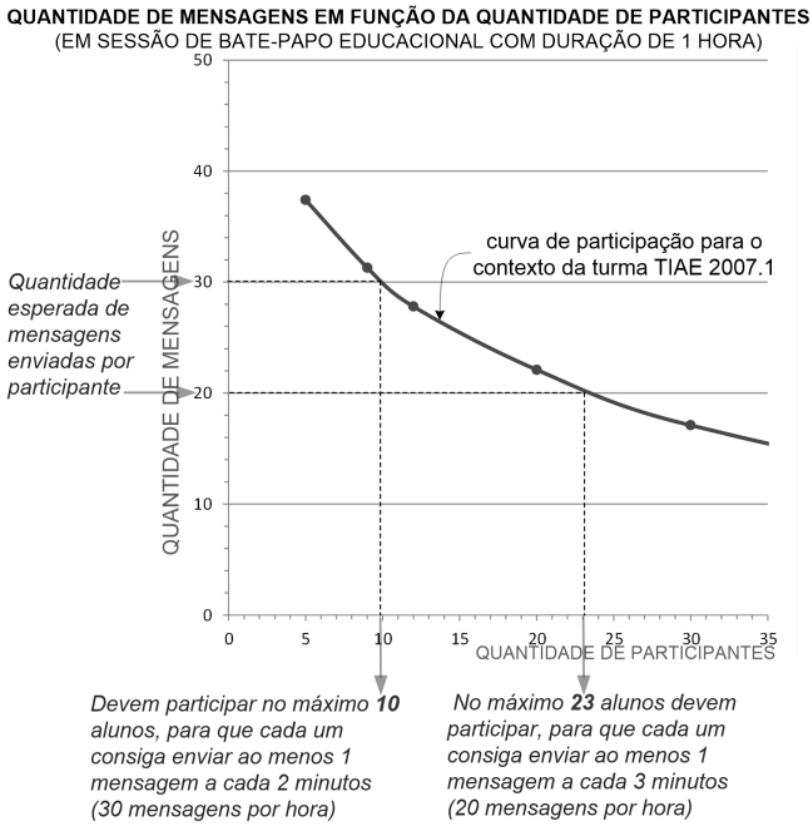

Figura 10: Quantidade de mensagens em função da quantidade de participantes para o contexto da turma TIAE 2007.1

Além do contexto específico de cada turma, o limite de alunos que podem participar da sessão de bate-papo educacional também depende da expectativa da quantidade de mensagens que cada aluno deve produzir durante a sessão. No exemplo apresentado na Figura 10, suponha que se espere que cada aluno envie, em média, 1 mensagem a cada 3 minutos de conversa, isto é, 20 mensagens durante uma sessão de 60 minutos. Pela curva identificase que esse mínimo de mensagens por participante é alcançado quando se tem, no máximo, 23 participantes. Caso o nível de participação esperado seja de 1 mensa- gem a cada 2 minutos ( 30 mensagens por hora), naquela turma deveriam participar no máximo 10 alunos da sessão de bate-papo.

A estimativa precisa requer que sejam obtidos os parâmetros discutidos nesse artigo, como o tempo médio de leitura e de digitação dos alunos, no contexto que se deseja estimar, para cada turma específica. Contudo, conforme ilustrado na Figura 11, estudando as várias turmas de uma disciplina é possível obter uma curva média que serve para estimar a produção média de mensagens em qualquer turma daquela disciplina. Essa é uma curva útil para definir a quantidade de participantes que podem participar das sessões de bate-papo naquela disciplina (independentemente do contexto).

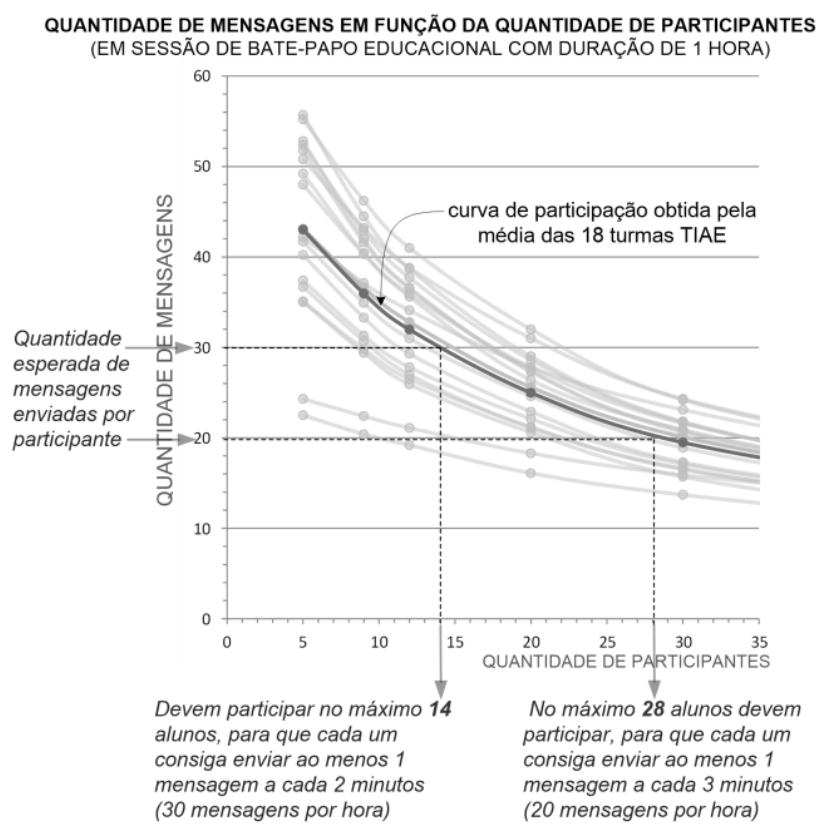

Figura 11: Curva média de participação nas turmas TIAE

Em trabalhos futuros, podem ser obtidos dados para se estimar a curva média de outras disciplinas de um mesmo curso, e tirar a média das disciplinas para se estimar a quantidade de alunos que devem participar de uma sessão de bate-papo em qualquer disciplina daquele determinado curso. E assim, generalizando cada vez mais, talvez seja possível obter uma estimativa média para as sessões de bate-papo de qualquer curso de nível superior, contudo ressaltamos que essa seria uma estimativa muito grosseira.

A partir dos resultados obtidos com a presente pesquisa, é possível discutir os resultados encontrados na literatura apresentados na seção 2. Não é possível enunciar um valor consensual sobre a quantidade de alunos que devem participar de uma sessão de bate-papo, pois depende do contexto de cada turma e da expectativa do professor sobre a produção de mensagens dos alunos ao longo da sessão de bate-papo. Os valores que encontrados na lite- 
ratura foram obtidos de contextos específicos e decorrentes da expectativa dos professores/pesquisadores sobre a participação dos alunos.

O que se pôde concluir, em função dos estudos aqui apresentados sobre as sessões de bate-papo das turmas de uma disciplina em particular, foi: não é preciso constituir grupos muito pequenos de apenas 3 ou 4 alunos, como sugerem alguns autores; mas também não devem ser agrupados muitos alunos (os pesquisadores das turmas estudadas sugerem evitar grupos com mais de 18 alunos [11]) porque a confusão começa a se tornar crítica: os alunos não conseguem ler todas as mensagens; a produção individual de mensagens decai; e sobra pouco tempo para o aluno refletir sobre o que está sendo discutido na sessão.

\section{Conclusão e Trabalhos Futuros}

No presente artigo foi elaborado um modelo matemático para representar o comportamento de usuários em sistemas de bate-papo no contexto educacional visando determinar qual o número máximo de alunos que devem participar de uma sessão de bate-papo. Os resultados produzidos pelo modelo elaborado mostraram-se adequados em comparação com os dados reais obtidos de 142 sessões de bate-papo realizadas em 18 turmas de uma disciplina a distância, e assim conclui-se que o modelo elaborado nessa pesquisa produz estimativas adequadas. O modelo é útil para subsidiar diferentes estudos, e nesse artigo mostrou-se a utilidade do modelo para apoiar o planejamento do tamanho do grupo de alunos que devem participar de uma sessão de bate-papo educacional de uma determinada disciplina - esse resultado é de interesse para as instituições que promovem educação online.

O presente artigo também contribuiu para um maior entendimento sobre a participação em bate-papo educacional. Buscou-se caracterizar o comportamento de participação em função de processos elementares: leitura e escrita de mensagens, e de reflexão. Foi possível equacionar o limite máximo de produção de mensagens em função da quantidade de participantes e, a partir do modelo elaborado, foi possível estimar a quantidade esperada dessa produção de mensagens num dado contexto.

Dentre as limitações dessa pesquisa estão os poucos dados obtidos dos experimentos sobre os processos elementares de participação - espera-se que os processos de leitura, escrita e reflexão sejam caracterizados com mais precisão e generalização em trabalhos futuros. Outra limitação da presente pesquisa é a concentração dos dados reais do corpus de análise estarem ao redor de 10 alunos por sessão, com desvio padrão de 3,8 alunos. Essa concentração impossibilitou a avaliação do modelo em grupos pequenos (com 5 ou menos alunos) e também em grupos muito maiores (com 20 ou mais alunos) - em trabalhos futuros, espera-se avaliar o modelo considerando uma maior variação no número de participantes em um mesmo contexto. Também espera-se, em trabalhos futuros, que sejam produzidas estimativas em outros contextos, variando-se as disciplinas e os cursos, visando obter uma estimativa genérica, ainda que grosseira, para as diferentes turmas de diferentes cursos de nível superior.

\section{Referências}

[1] INEP. Censo de Educação Superior. Disponível em

$<$ http://portal.mec.gov.br/index.php?option=com _docman\&task=doc_download\&gid=9332\&Ite $\overline{\mathrm{mid}}=>$. Acessado em 18 ago 2012.

[2] CENSOEAD.BR. Relatório analítico da aprendizagem a distância no Brasil. Associação Brasileira de Educação a Distância. São Paulo: Pearson Education do Brasil, 2010.

[3] O. Böhlke. A comparison of student participation levels by group size and language stages during chatroom and face-to-face discussions in German. In CALICO Journal, 21 (1), páginas 67-87, 2003.

[4] A. Caspi, P. Gorsky, E. Chajut. The influence of group size on non-mandatory asynchronous instructional discussion groups. In The Internet and Higher Education. 6(3), páginas 227-240, 2003.

[5] S. Hrastinski. Participating in synchronous online education. Doctoral dissertation, Lund University, 2007.

[6] A. Löber, G. Schwabe, S. Grimm. Audio vs. chat: The effects of group size on media choice. Proceedings of the 40th HICCS Hawaii International Conference on System Sciences, 2007.

[7] L. Kleinrock. Queueing Systems - Theory, Vol. I. Wiley-Interscience, 1975.

[8] M. Pimentel, M. A. Gerosa, H. Fuks. Sistemas de comunicação para colaboração. In Sistemas Colaborativos, cap.5. Rio de Janeiro: Elsevier, 2011.

[9] L. D. Calvão, M. Pimentel, H. Fuks. Do email ao Facebook. Uma perspectiva evolucionista sobre os meios de conversação da internet. Rio de 
Janeiro: UNIRIO, 2014.

[10] J. G. Hilgert. A construção do texto "falado" por escrito na Internet. In Fala e escrita em questão. Dino Preti (org). São Paulo: Humanistas/FFLCH/USP, 2000.

[11] M. Pimentel, C. J. P. Lucena, H. Fuks. "Debati, debati... aprendi? Investigações sobre o papel educacional das ferramentas de bate-papo". In IX Workshop sobre Informática na Escola WIE2003. Agosto, 2003.

[12] M. Silva. Avaliação da Aprendizagem em Educação Online. 2010. Entrevista publicada no YouTube: <http://youtu.be/S7uUd6afEYE>, Acessado em 12 ago 2013.

[13] Referenciais de qualidade para educação superior a distância. MEC/SEAD, Brasília, 2007.

[14] S. Hrastinski. What is online learner participation? A literature review. Computer \& Education, 2008.

[15] G. Stahl. Studying Virtual Math Teams. New York, NY: Springer, 2009.

[16] H. Fuks, M. Pimentel, C.J.P. Lucena. R-UTyping-2-Me? Evolving a chat tool to increase understanding in learning activities. In International Journal of Computer-Supported Collaborative Learning, v. 1, p. 117-142, 2006.

[17] M. H. Beauvois. Computer-assisted classroom discussion in the foreign language classroom: Conversation in slow motion. In Foreign Language Annals, 25, 455-464, 1992.

[18] J. Bump. Radical changes in class discussion using networked computers. In Computer and the Humanities, 24, 49-65, 1990.

[19] S. Hrastinski. The potential of synchronous communication to enhance participation in online discussions: A case study of two elearning courses. In Information \& Management, 45, 499-506, 2008.

[20] D. J. Piva, R. Pupo, L. Gamez, S. Oliveira. EAD na Prática - Planejamento, métodos e ambientes de educação online. Elsevier Editora Ltda. páginas 164-170, 2011.

[21] R. Gentleman, R. Ihaka. The R Project for Statistical Computing. <http://www.r-project.org/>, Acessado em 10 ago 2013.

[22] S. Shimakura. CE003 - Estatística II. $<$ http://leg.ufpr.br/ silvia/CE003/> , acessado em 20 jul 2013., 24, 49-65, 1990.
[23] R. Gaines, W. Lisowski, S. Press, and N. Shapiro. Authentication by keystroke timing: Some preliminary results. Rand Report R-256NSF. Rand Corporation, Santa Monica, CA, 1980.

[24] J. Garcia. Personal identification apparatus. Patent Number 4.621.334. U.S. Patent and Trademark Office, Washington, D.C., 1986.

[25] E. De Souza e Silva, D. Figueiredo, R. Leão. The TangramII integrated modeling environment for computer systems and networks. In SIGMETRICS Perform. Eval. Rev., 36(4):64-69, 2009.

[26] M. C. Fogliatti, N. M. C. Mattos. Teoria de filas. Rio de Janeiro: Interciência, 2007.

[27] H. Fuks, M. A. Gerosa, C. J. P. Lucena. "The Development and Application of Distance Learning on the Internet". In The Journal of Open and Distance Learning, Vol. 17, N. 1, ISSN 0268-0513, pp. 23-38, 2002.

[28] J. Wainer. Métodos de pesquisa quantitativa e qualitativa para a Ciência da Computação, em JAI 2007 - Jornada de Atualização em Informática, Anais do XXVII Congresso da Sociedade Brasileira de Computação. p.221-262, 2007. 